\title{
Trends and Outcomes of Emergency Pediatric Surgical Admissions from a Tertiary Hospital in Ethiopia
}

\author{
Temesgen Firomsa ${ }^{1}$, Muluwork Teferra ${ }^{2}$, Amezene Tadesse ${ }^{3 *}$
}

\footnotetext{
OPEN ACCESS

Citation: Temesgen Firomsa, Muluwork Teferra, Amezene Tadesse. Trends and Outcomes of Emergency Pediatric Surgical Admissions from a Tertiary Hospital in Ethiopia. Ethiop J Sci.2018;28(2):251.

doi:http://dx.doi.org/10.4314/ejhs.v28i3.2

Received: August 29, 2017

Accepted: September 8, 2017

Published: May 1, 2018

Copyright: (C) 2018 Temesgen Firomsa., et al. This is an open access article distributed under the terms of the Creative Commons Attribution License, which permits unrestricted use, distribution, and reproduction in any medium, provided the original author and source are credited. Funding: Nil

Competing Interests: The authors declare that this manuscript was approved by all authors in its form and that no competing interest exists.

Affiliation and Correspondence: ${ }^{1}$ Department of Pediatrics and Child Health, School of Medicine, Addis Ababa University, Ethiopia

${ }^{2}$ Department of Pediatrics and Child Health and Pediatric Emergency Consultant, School of Medicine, Addis Ababa University, Ethiopia

${ }^{3}$ Department of surgery, School of Medicine, Addis Ababa University, Ethiopia

*Email: amezenet@yahoo.com
}

\section{ABSTRACT}

BACKGROUND: Pediatric surgical emergency condition is a broad spectrum of surgical pathologies/disorders occurring in pediatric age group requiring proper emergency surgical care as the only option of management to salvage life, avoid/minimize disability or palliation. The objective of this study is to analyze the pattern and outcome of pediatric surgical emergencies managed at Tikur Anbesa Hospital and the factors associated with outcome.

METHODS: Retrospective analysis of medical records of patients was conducted to assess the pattern, outcome and factors associated with outcome of pediatric surgical emergencies admitted and managed at Tikur Anbesa Specialized Hospital, from January 1, 2015 to December 31, 2015. Data was checked for completeness and entered into SPSS version 20 for statistical analysis.

RESULTS: A total of 454 patients' medical records were analyzed. The presentation was after 5 days of illness in 182(40\%), and $>3$ days in 243(53.4\%), while only 89(19.6\%) of them come within 24 hours of the illness. The etiologies were abdominal/gastrointestinal emergencies 210(46.3\%), foreign body in aero digestive system in 133(29.3\%), urogenital surgical emergencies in $27(5.9 \%)$. The other causes include superficial and deep tissue abscess collections in 22(4.8\%), trauma /injury related cases in 24(5.3\%) and others in 38(8.4\%) respectively while congenital malformations were only 57(12.6\%) of the cases. Finally, 439(96.7\%) were discharged cured or improved without major disabilities documented, while 9(2\%) died.

CONCLUSIONS: Delayed presentation, unstable condition on arrival, younger age group and the nature of underlying pathology could have contributed for the death, but this needs further study.

KEYWORDS: Pediatric surgery, Emergency Surgery, Ethiopia

\section{INTRODUCTION}

Pediatric surgical emergency condition is a broad spectrum of surgical pathologies/disorders occurring in pediatric age group requiring proper emergency surgical care as the only option of management to salvage life, avoid/minimize disability or palliation $(1,2)$. These conditions include a wide range of surgical disorders with variable incidences in different socio-demography, age and time. 
In general acquired or congenital abdominal surgical emergency (appendicitis, intussusception, IHPS, incarcerated hernias, intestinal atresia or stenosis, HSD with obstruction,...etc.) are the commonest causes of surgical emergency in pediatrics. Trauma, congenital anomaly affecting other systems, aero-digestive foreign bodies, infectious (abscesses, intestinal parasitosis, croup...etc.), urologic emergencies (acute obstructive uropathies, testicular torsion, etc.) are the other ones. Neoplastic and other conditions requiring urgent surgical intervention also contributes for significant mortality and morbidity in children $(1,2,3,4,5)$.

Surgically treatable conditions are important public health problem in low and medium income countries, and the magnitude of surgically avertable burden exceeds the burden of some of the most widely recognized global health problems. According to global disease burden, surgical conditions in general are responsible for approximately $6-12 \%$ of all pediatric admissions in sub-Saharan Africa, although the proportion may be higher in some urban areas (6).

Although knowledge regarding the magnitude, pattern, challenges and outcome of pediatric surgical emergencies in particular is inadequate in sub-Saharan countries including Ethiopia, few reports from tertiary teaching hospitals and zonal hospitals in these countries suggest surgical emergencies in pediatrics are major unmet health problem, contributing to the majority of pediatric surgical admissions and increased mortality $(4,7,8)$.

Emergency surgical conditions/diseases in pediatrics need proper and timely surgical and perioperative supportive care than elective surgery or the same pathology in adults. These delicate characteristics of the diseases coupled with disproportionately increased burden of the cases in tertiary hospitals in developing countries like Ethiopia where common general surgical cases are managed barely or not at all at district hospitals, further compromises the service provision. Besides the limited human resources and poor infrastructure have a negative impact to service rendering. Consequently, the mortality and morbidity are exponentially higher compared to high income countries.

Policy makers have misconceptions that quality surgical care is cost prohibitive and too complex to be seriously considered a viable conduit for improving global health (9). In addition to many socio-economic factors contributing to the disparity in access to surgical care, lack of information about the burden of surgical disease and surgery's impact on communities has led surgical service to be neglected.

In Ethiopia, the available studies are institution based reviews from TASH (11 years ago), Jimma University Hospital (from February 2010 to November 2011) and Yirgalem Zonal Hospital (September 2004-August 2005). These reviews showed that surgical conditions in pediatrics constitutes $33 \%, 20 \%$ and $10 \%$ of the total pediatric admissions respectively, while emergency conditions accounts for $50 \%$ to $78 \%$ of the cases. The mortality is significantly higher in emergency conditions, even all deaths being exclusively emergency cases from the latter study $(4,8,10)$.

Tikur Anbesa Specialized Hospital (TASH) has high burden of patient flow. Under five surgical emergencies are deferred even by many referral hospitals found in the Addis Ababa due to anesthesia and other reasons. As a result, the hospital is obligated to manage all cases including common general surgical emergencies which could have been managed at general hospitals.

Therefore, knowing the current information regarding the burden, character and type of common surgical emergencies along with the factors affecting the outcome in TAH is crucial.This can be the first step in strategies of improving the care. It can also help as a clue for further studies at different level. Therefore, conducting a study on this area is required to narrow the information gap specifically regarding current surgical emergencies in pediatrics at our institution.

Additionally, the findings of this study are hoped to provide some knowledge and insight into the prevalence, risk factors and outcome of

DOI: http://dx.doi.org/10.4314/ejhs.v28i3.2 
the condition at study area. It could also form the basis for further research.

\section{MATERIALS AND METHODS}

The study is institutional based and was conducted in Tikur Anbesa Specialized Hospital (TASH), Addis Ababa, Ethiopia. TASH is the largest and the most overcrowded specialized hospital in the country. It is the teaching hospital of Addis Ababa University. Emergency and elective pediatric surgery are the major services provided by the institution for patients referred from all over the country. The hospital has about 187 beds for pediatrics in general excluding the neonatal ward.

Study design: Institution based retrospective descriptive study was conducted between January 1 and December 31, 2015 to assess the pattern, outcome and factors associated with outcome of pediatric surgical emergencies admitted and managed at TASH.

Study population: The study population included all pediatric patients admitted for surgical emergency conditions during 12 months of the year 2015.

Inclusion criteria: All patients aged 7 days -12 years, diagnosed and managed for emergency surgical diseases at Tikur Anbesa Teaching Hospital Pediatric Unit were included in the study.

Exclusion criteria: Neonates less than 7 days old on presentation/admission were not included as these are admitted and managed at neonatal intensive care unit. Pediatric surgical cases who needed cardiac, orthopedic and neurosurgical intervention. Surgical services for ophthalmologic and plastic and reconstructive cases are not available in TASH.

Data collection instrument and procedure: The target study populations were first identified from registration books in the wards and operation theatre. Then the patients' medical records were retrieved and data was collected using a preprepared standard and structured questionnaires from the chart.

Data entry and analysis: The collected data was checked for completeness, coded, entered into SPSS version 20 statistical software packages, cleaned and analyzed. During analysis, descriptive statistics of frequency and percentage were used, and the outputs displayed through tables.

Data quality assurance: During preparatory stage, the questionnaires were carefully designed, pre-coded and pretested to minimize errors.. During data entry and clearance, error was minimized by using trained persons. During data analysis and report writing, appropriate statistical technique was used.

Ethical consideration: Ethical clearance was obtained from the Research and Publication Committee of the Department of Pediatric and Child Health, School of Medicine, Addis Ababa University. No personal identifications were included in the questioner. Confidentiality of all the data was kept throughout the different stages of the study.

\section{RESULTS}

Out of 503 cases of pediatric surgical emergencies retrieved from admission and operation theatre registration books, medical records of 478 were found. Twenty four charts with incomplete records were excluded. Finally 454 patients' medical records were analyzed.

In this study, the majority of the patients, $328(72.2 \%)$, were in the age group of 3-5 years, while $7616.7 \%$ ) were 5 years old or above while $50(11.1 \%)$ were less than 3 years of age. Of all, $302(66.5 \%)$ were males and $152(33.5 \%)$ were females with a male-to-female ratio of $2: 1$. The majority of the patients were from Addis Ababa (AA) and Oromia, 221(48.7\%) and 152(33.5) respectively (Table 1 ).

The majority of the cases were referred by zonal/higher hospital, 251(55.3\%), and district/primary hospital, $81(17.8 \%)$. Most of the cases, 348(76.7\%), were referred for management purpose after diagnosis was made. While the rest, $106(23.3 \%)$, for both diagnoses and intervention The commonest presentations seen in 201(44.3\%) of the cases were nonspecific gastrointestinal symptoms (including abdominal pain and/or diarrhea with or without vomiting), followed by history suggestive of foreign body in aero

DOI: http://dx.doi.org/10.4314/ejhs.v28i3.2 
digestive system, 113(24.9\%), and others (Table 2).

Table1: Socio-demographic characteristics

\begin{tabular}{llll}
\hline Variables & Category & Frequency & $\mathbf{\%}$ \\
\hline Age & $<$ 3years & 50 & 11.0 \\
& 3-5years & 328 & 72.2 \\
& $>$ 5years & 76 & 16.7 \\
& Total & 454 & 100 \\
\hline Sex & Male & 302 & 66.5 \\
& Female & 152 & 33.5 \\
& Total & 454 & 100 \\
\hline Address & Addis Ababa & 221 & 48.7 \\
by region & Oromia & 152 & 33.5 \\
& Amhara & 37 & 8.1 \\
& Tigray & 3 & .7 \\
& SNNP & 32 & 7.0 \\
& Other & 9 & 2.0 \\
& Total & 454 & 100.0 \\
\hline
\end{tabular}

The patients' specific diagnoses categorized under urogenital surgical emergencies were testicular torsion, post-circumcision complication, obstructive uropathy secondary to PUV, bladder outlet obstruction secondary to hypospadias with meatal stenosis, bladder stone and others. The congenital anomalies presented with intestinal obstruction include anorectal malformations with or without fistula, anal stenosis, Hirschsprung's diseases, incarcerated inguinal hernia, jejunal atresia and others (Table 3).

In $182(40 \%)$ of the cases, the duration of illness is more than 5 days on presentation to our hospital while 243(53.4\%) arrived after more than 3 days of the onset of illness. Only $89(19.6 \%)$ of them come within 24 hours of the illness. The condition on arrival was stable in $283(62.3 \%)$ of the cases. The modes of intervention were operative surgical intervention in $308(67.8 \%)$, non-operative procedures in $114(25.1 \%)$ and conservative in $32(7 \%)$ (Table 4). Out of 454 patients, 439(96.7\%) were discharged cured or improved without major disabilities documented. Nine patients (2\%) died (Table 5) and 6 patients discharged with comorbidities (including tracheostomy, limb deformity, HTN). The mean hospital stay was 4.8 days.

Table 2: Presenting complaints of the study participants

\begin{tabular}{lll}
\hline Presenting complaints & Frequency & Percentage \\
\hline Abdominal pain, or distension, and/or diarrhea/vomiting & 201 & 44.3 \\
Failure to pass feaces/flatus and abdominal distension & 18 & 4.0 \\
Foreign body swallow & 67 & 14.8 \\
History of chocking episode or witnessed aspiration & 46 & 10.1 \\
Cough and SOB & 32 & 7.0 \\
Failure to pass urine or difficulty of micturition & 19 & 4.2 \\
Trauma history/injury & 23 & 5.1 \\
Others & 48 & 10.6 \\
Total & 454 & 100.0 \\
\hline
\end{tabular}

DOI: http://dx.doi.org/10.4314/ejhs.v28i3.2 
Table 3: Final diagnosis

\begin{tabular}{lll}
\hline Final diagnosis & Frequency & Percent \\
\hline Appendicitis & 58 & 12.8 \\
Foreign body aspiration & 61 & 13.4 \\
Foreign body swallow & 72 & 15.9 \\
Intussusception & 63 & 13.9 \\
IHPS & 40 & 8.8 \\
\hline Intestinal obstruction secondary to congenital anomalies & \\
\hline ARM & 7 & 1.5 \\
Anal stenosis & 4 & 0.9 \\
HSD & 15 & 3.3 \\
Incarcerated inguinal hernia & 5 & 1.1 \\
Others & 8 & 1.8 \\
\hline Urogenital emergencies & & \\
\hline Testicular torsion & 5 & 1.1 \\
Post circumcision complication & 3 & 0.66 \\
Hypospadias with meatal stenosis & 4 & 0.9 \\
Obstructive uropathy secondary to PUV & 9 & 2 \\
Bladder outlet obstruction secondary to & 4 & 0.9 \\
bladder stone & & \\
Other & 3 & 0.66 \\
\hline Abscess collection & 22 & 4.8 \\
Trauma/injury & 24 & 5.3 \\
Others & 48 & 10.6 \\
Total & 454 & 100.0 \\
\hline
\end{tabular}

Table 4: The duration of illness, condition on arrival, intervention done and outcome.

\begin{tabular}{llcc}
\hline \multirow{3}{*}{$\begin{array}{l}\text { Duration of illness } \\
\text { on arrival }\end{array}$} & Variables & Frequency & Percentage \\
\cline { 2 - 4 } & 24 hrs & 89 & 19.6 \\
& $49-72$ & 82 & 18 \\
& $3-5$ days & 40 & 8.8 \\
& $>5$ days & 61 & 13.4 \\
Condition on arrival & Unstable & 182 & 40 \\
& Stable & 171 & 37.7 \\
\hline Outcome & Discharged improved & 283 & 62.3 \\
& Died & 439 & 96.7 \\
& Disabilities/morbidities & 9 & 1.98 \\
Surgical & Operative & 6 & 1.3 \\
intervention & Non operative & 114 & 67.8 \\
& Conservative & & 25.1 \\
& management & 32 & 7.0 \\
\hline
\end{tabular}

DOI: http://dx.doi.org/10.4314/ejhs.v28i3.2 
Table 5: Lists of Deaths, diagnosis, duration of illness and modes of intervention

\begin{tabular}{|c|c|c|c|c|c|}
\hline & Diagnosis & Frequency & Age & $\begin{array}{l}\text { Duration of } \\
\text { illness }\end{array}$ & Intervention done \\
\hline 1 & Intussusception & 3 & $\begin{array}{l}1,4 \& \text { 5months } \\
\text { resp. }\end{array}$ & $\begin{array}{l}>6 \text { days } \\
\& 10 \text { days }\end{array}$ & $\begin{array}{l}\text { Only one died } \\
\text { postop }\end{array}$ \\
\hline 2 & HSD & 2 & $\begin{array}{l}\text { 12days and } \\
1 \text { month }\end{array}$ & since birth & Operated \\
\hline 3 & Congenital lobar & 1 & 2 months & 45days & Not operated \\
\hline 4 & Jejunal atresia & 1 & 13days & 13days & Operated \\
\hline 5 & TEF & 1 & 16days & 16days & Operated \\
\hline 6 & Mid-gut volvulus & 1 & 15days & 7days & Operated \\
\hline
\end{tabular}

\section{DISCUSSION}

This hospital based retrospective study assessed the pattern of pediatrics surgical emergency at TASH, Ethiopia. The result showed that among the 454 cases analyzed, a large proportion of participants, $328(72.2 \%)$, were in the age group from 3-5 years, followed by the age group greater than five $76(16.7 \%)$. This result is different as compared with a study done in Cairo in which age ranged between few hours and 12 years with a mean age of 11.7 month (11), but similar with another study done prospectively in 2005 on the pattern of pediatric surgical conditions at Yirgalem Zonal Hospital, Ethiopia. A total of 1389 children were admitted to the hospital with the average age being 48 months, out of which $144(10.36 \%)$ were admitted with surgical illnesses (8).

The study also showed male predominance with male to female ratio of $2: 1$. This is consistent with studies done in Yirgalem Hospital where male to female ratio of 2.8:1 (7), and Jimma Uiversity Hospital, done in 2010 with 2:1 (8).

There were a variety of specific etiologies/diagnoses seen in this study. The most leading causes are categorized under abdominal/gastrointestinal emergencies (Intussusception, Appendicitis, IHPS, Intestinal obstruction secondary to congenital anomalies) which constitute $210(46.3 \%)$ of the total, followed by aero digestive foreign body $133(29.3 \%)$, urogenital surgical emergencies 27(5.9\%), superficial and deep tissue abscess collections in $22(4.8 \%)$, trauma /injury related cases of $24(5.3 \%)$ and others $38(8.4)$ respectively.

Congenital anomalies were only 57(12.6\%) in this study, unlike the study conducted in Tanzania which showed them to be the leading cause of pediatric surgical emergencies, 47(43\%) (7). Except the foreign body in the aero digestive system which makes a significant proportion in ours, the finding is similar with the study done in Cairo: Intussusception, 169 cases (23.8\%), infantile hypertrophic pyloric stenosis, 107 cases $(15 \%)$, and anorectal anomalies, 66 cases $(9.2 \%)$ (11).

In TASH acute appendicitis was the commonest surgical condition seen in $729(12 \%)$ of the total surgical procedures (both emergency and elective) in five years (4). Though appendicitis accounts for 58(12.8\%) of the total surgical emergency admission as seen in this study, it seems the incidence of appendicitis in TASH is dropping. This is probably due to increased number of facilities managing the case over the last 11 years gap between the two studies. In Yirgalem, the leading cause of admission was found to be intussusceptions in $19(14.1 \%)$ (8), and in Jimma University Specialized Hospital, the most common diagnoses were trauma, 224(23.2\%), surgical infection, $202(21.0 \%)$, and gastrointestinal problems, 142(14.7\%) (10). The difference could be explained by the different setup with varying inservice provision capacities of hospitals.

DOI: http://dx.doi.org/10.4314/ejhs.v28i3.2 
The current study showed the overall mortality rate of $2 \%$ which is lower than the study conducted in Yirgalem Hospital (7.4\%) (8) and $3.4 \%$ in Cairo (11). The lower death rate in this study compared to study done at Yirgalem is expected because of the difference in the setup with difference in the quality and type of service provided. The better outcome recorded compared to the study done in Cairo could be due to age limitation in this study in which neonates of age less than 7 days were not included. As a result of which most congenital surgical emergencies with poor or limited outcome were excluded (12). The leading causes of death in this study were congenital anomalies in 6 of 9 deaths, while the rest 3 were intussusceptions. On the other hand, 4 of the 9 patients died before surgery during stabilization while 5 patients died in the postoperative period. All the cases who died arrived late (duration of illness was more than 5 days at admission), and 9 of them were younger than 6 months.

The mean hospital stay was 4.8 days, which was lower than the study one in Cairo, which was 5.7 days. Of all pediatric surgical emergencies admitted, 254(56\%) were general surgical emergencies (intussusception, appendicitis and aero digestive foreign body) which could have been managed at primary or zonal referral hospital.

This study has provided recent knowledge on pediatric surgical emergency at TASH. To our knoeledge, it is also the first study on pediatric surgical emergency in our setup and in our country.

Limitations of the study include:

$\checkmark$ The study design used (retrospective) limits the capacity to draw any causal inferences;

$\checkmark$ The sample size was not calculated scientifically; rather, all the emergency surgical admissions were included. This may affect the conclusions to be drawn from the research;

$\checkmark$ Incomplete documentation was a setback;

$\checkmark$ It did not include neonates of below 7 days old.
In conclusion, most of the cases in this study were uncomplicated general surgical emergencies, and the majority of them were referred from zonal or even higher level hospitals. This has resulted in overcrowding of TASH with cases which could have been handled by the other centers. There is a delay in presentation of the cases especially those who died. This could be due to unnecessary referral, poor health service seeking behavior and unavailability of expertise in the referring hospitals. This needs further investigation to rectify the problem and to reduce the morbidity and mortality associated with it.

\section{REFERENCES}

1. David J. Hackam, Tracy Grikscheit, Kasper Wang, Jeffrey S. Upperman, and Henri R. Ford, Raymond R. Price and Catherine R. deVries. Introduction to pediatric surgery, global burden of surgery. In Schwartz's Principles of Surgery.10th edition. chapter 39:1597-1600 \& chap;49;1955-78.

2. S.W. Bickler, H. Rode. Surgical services for children in developing countries. Bull World Health Organ [online] 2002; 80(10):829-835. ISSN 0042-9686.

http://dx.doi.org/10.1590/S004296862002001000013.

3. S.W Bickler, Sanno-Duanda B. Epidemiology of pediatric surgical admissions to a government referral hospital in the Gambia. Bull of the World Health Organ 2000; 78(11):1330-6.

4. Derbew M, Ahmed E. The pattern of pediatrics surgical conditions inTikur Anbessa Hospital, Addis Ababa, Ethiopia. Ethiop Med J, 2006;44:331-338.

5. Sewagegn Yeshiwas, Damte Shimelis. Patterns of Foreign Body in the AeroDigestive Tract of the Pediatric Patients in Tikur Anbessa Hospital. Ethiopian journal of Pediatrics and Child Health, 2010;(6): 4-13.

6. S.W. Bickler, Thomas G. Weiser, Nicholas Kassebaum, et al. Global Burden of Surgical Conditions, disease control priorities. In Essential surgery, volume 1: 3rd ed.:19-40.

DOI: http://dx.doi.org/10.4314/ejhs.v28i3.2 
7. Penoyar $\mathrm{T}$, Cohen $\mathrm{H}$, Kibatala $\mathrm{P}$, et al. Emergency and surgery services of primary hospitals in the United Republic of Tanzania. BMJ Open 2012;2: e000369. doi: 10.1136/bmjopen-2011-000369.

8. Tekle TT, Mollalegne TM. Pattern of Pediatric Surgical Admission in Yirgalem Hospital Southern Ethiopia. J Vasc Med Surg 2015; 4: 239 . doi:10.4172/23296925.1000239 .

9. Stephen W. Bickler. Global Burden of Surgical Disease. In The Lancet Commission on Global Surgery, Boston, MA January 16, 2013.
10. Wondafrash Y. Pediatric surgery in Jimma University specialized hospital, (from February 2010 to November 2011): [NON153].

11. Ahmed Elham, Ahmed Fares,Magdy El Barbary, Assem El Fiky, Naser Abdelal. Pattern and incidence of pediatric surgical emergencies in Cairo university children hospital, Giza, Egypt. Master (Msc) Thesis, 2001.

12. EndaleTefera, TelahunTeka, Milliard Derbew. Neonatal gastrointestinal surgical emergencies: a 5- year review in a teaching hospital in Addis Ababa, Ethiopia. Ethiop Med J 2007; 45( 3):251-6. 